Sitientibus Série Ciências Físicas 16, sf01609-15 (2020)

\title{
Estudo Espectroscópico do Par de Galáxias Interagentes ESO 505-IG 030 e ESO 505-IG 031
}

\author{
Spectroscopic Study of the \\ Pair of Interacting Galaxies ESO 505-IG 030 and ESO 505-IG 031
}

\author{
Elis Cristina Silva Sales e Vera Aparecida Fernandes Martin, Paulo César da Rocha Poppe, \\ José Henrique Costa Pinto Souza, Mateus do Carmo Carvalho, Toni Cordeiro de Almeida, \\ Raquel Silva Freitas, Bianca Ramielly Bomfim de Jesus, Mariana da Silva Lima Santos \\ Departamento de Física - UEFS \\ Av. Transnordestina, $s / n$, Novo Horizonte, \\ Feira de Santana - BA - 44036-900
}

\begin{abstract}
Este trabalho apresenta os resultados das observações espectroscópicas de fenda longa realizadas para o par de galáxias peculiares interagentes ESO 505-IG 030 e ESO 505-IG 031. Os dados foram obtidos com o espectrógrafo Cassegrain acoplado ao telescópio Perkin-Elmer $(1,60 \mathrm{~m})$ do Observatório do Pico dos Dias/Laboratório Nacional de Astrofísica (OPDLNA/MCTI). Trata-se de um par interagente no Universo local $(z \approx 0,03957)$ classificadas como espirais do tipo morfológico Sc. Curvas de rotação, síntese espectral e alguns parâmetros físicos e geométricos são apresentados e discutidos para cada um dos objetos estudados.
\end{abstract}

Palavras-chaves: galáxias peculiares; espectroscopia; interação gravitacional.

This work presents the results of the long-slit spectroscopic observations carried out for the pair of peculiar interacting galaxies ESO 505-IG 030 and ESO 505-IG 031. The data were obtained with the Cassegrain spectrograph coupled to the 1.60-m Perkin-Elmer telescope of the Pico dos Dias Observatory/National Astrophysics Laboratory (OPD-LNA/MCTIC). It is an interacting pair in the local Universe $(z \approx 0.03957)$ classified as spirals of the Sc morphological type. Rotation curves, spectral synthesis and some physical and geometric parameters are presented and discussed for each of the objects studied.

Keywords: peculiar galaxies; spectroscopy; gravitational interaction.

\section{INTRODUÇÃO}

A História da Astronomia revela que as observações realizadas no século XVIII indicavam a presença de objetos diferentes, caracterizados como "nuvens" extensas, difusas e luminosas, que foram registrados, inicialmente, com a mesma denominação de "nebulosas" [1]. Estes novos objetos receberam um rápido destaque por parte dos astrônomos e com a constante melhora dos telescópios, dos métodos de detecção e das técnicas observacionais, o início do século XX já oferecia para a comunidade científica a compilação de catálogos com

*E-mail: eliscris.ssales@gmail.com cerca de 15 mil dessas "nebulosas", onde algumas já haviam sido corretamente identificadas como aglomerados estelares ou como nebulosas gasosas. Contudo, havia uma parcela significativa desses objetos, as "nebulosas espirais", que permaneciam com a natureza observacional sem uma adequada ou correta explicação.

Naquela época, os limites da Via Láctea não eram totalmente estabelecidos e o problema crucial estava na dificuldade de determinar as distâncias dos objetos "nebulosos" até então observados. Não havia um consenso formado entre os astrônomos e a resposta se tais objetos pertenciam, ou não, a Via Láctea, estava completamente aberta. Nesse contexto de incerteza, argumentações científicas eram externadas na tentativa de elucidar o problema, de 
modo que haviam defensores sustentando duas contraditórias hipóteses: de um lado Harlow Shapley (1885-1972) do Observatório Monte Wilson, argumentando que as nebulosas espirais eram objetos da própria Galáxia e, do outro lado, Heber Doust Curtis (1872-1942) do Observatório de Lick, sustentando a ideia contrária, ou seja, de que eram objetos dis- tantes, fora do domínio da Via Láctea. Tal problema alcançou tamanha repercussão que resultou no famoso debate em 26 de Abril de 1920 no Smithsonian Museum de História Natural, mas, infelizmente, sem nenhum resultado ou solução conclusiva para o problema, tornando-o, apenas, um registro histórico para os anais da Astronomia.

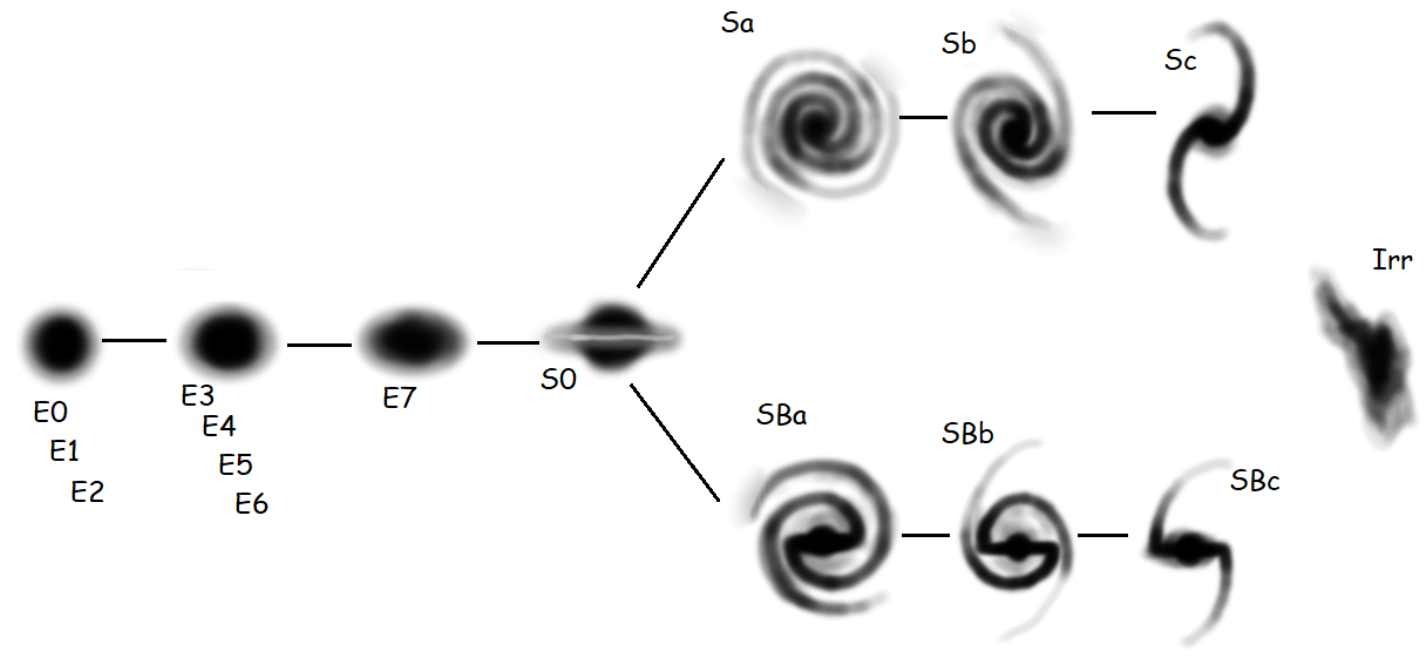

FIGURA 1: Esquema de classificação atual das galáxias a partir das observações de Hubble. As espirais são subdivididas em duas famílias: barradas e não-barradas. É importante ressaltar de que o arranjo acima não caracteriza uma sequência evolutiva desses objetos.

O calor da discussão sobre a natureza desses objetos não teve tempo para arrefecer e, em 1923, o astrônomo norte-americano Edwin Powell Hubble (1889-1953) conseguiu a partir de um telescópio de $2,5 \mathrm{~m}$ de diâmetro recém instalado no Observatório Monte Wilson, onde Shapley havia deduzido em $1914 \mathrm{com}$ um telescópio de $1,5 \mathrm{~m}$ que o Sol estava localizado perto do plano central da Via Láctea, e não no centro, cerca de 30 mil anos-luz de distância, detectar e medir as estrelas individuais (variáveis do tipo Cefeidas [2]) na nebulosa espiral Andrômeda [3] (M31), o que levou a determinação das respectivas distâncias e a resposta conclusiva para o problema até então aberto, ou seja, de que tais objetos não perten- ciam (eram externos) à Via Láctea. Portanto, como conclusão, o fato direto de que esta não representava a única galáxia existente no Universo. Esse resultado, por assim dizer, inaugurava o campo da Astronomia Extragaláctica e marcava o início da Cosmologia, que busca explicar a origem e evolução do Universo a partir da distribuição e movimento das galáxias.

As observações de Hubble no Monte Wilson seguiram a todo vapor e revelaram que as galáxias diferiam bastante entre si. Mas, curiosamente, a grande maioria apresentava, quando observadas em projeção contra o céu, aspectos morfológicos muito parecidos, que poderiam sugerir um agrupamento em famílias ou em classes. Esses objetos seriam as galáxias 
elípticas e as galáxias espirais. Alguns outros objetos, entretanto, apresentavam um aspecto intermediário entre essas duas classes e foram denominadas de lenticulares (galáxias S0 [4]). Havia, ainda, aquelas galáxias que fugiam completamente desses aspectos morfológicos (elípticas, espirais e lenticulares) e foram agrupadas em uma classe particular chamada de irregulares, sem simetrias de rotação ou estruturas bem definidas. São subdivididas em dois grupos bem distintos: Irr I (facilmente resolvidas em estrelas do tipo OB e regiões H II; como exemplo, as nuvens de Magalhães) e Irr II (não podem ser resolvidas em estelas; um exemplo típico é a galáxia M82).

Como resultado observacional deste estudo, Hubble organiza e publica um dos primeiros e mais simples esquemas de classificação de galáxias, usado até hoje, onde as mesmas são dispostas de acordo com a morfologia observada [5. A Figura 1 ilustra um esquema dessa classificação.

Seguindo o curso das observações, os estudos posteriores iriam revelar que, ao contrário do que se imaginava, as galáxias não estão distribuídas de modo aleatório no Universo. Elas se encontram relativamente próximas entre si e são as unidades básicas formadoras das estruturas em grande escala do Universo, organizando-se em aglomerados e superaglomerados, onde estão sujeitas ao processo de expansão e da interação gravitacional mútua [626. O processo de interação (fusão, colisão ou efeito de maré) tem-se mostrado mais comum do que o esperado, de tal forma que alguns dos tipos de galáxias que observamos atualmente, por exemplo, as galáxias peculiares [6, 7, podem ter tido sua origem parcial ou total no fenômeno de interação e não apenas na evolução dinâmica do objeto em um dado estado isolado. De certa forma, essa análise implica (ou sugere) em reexaminar o nosso ponto de vista sobre a classificação e evolução das galáxias.

O presente trabalho apresenta os resultados espectroscópicos obtidos ao longo de um estudo de Iniciação Científica que envolve uma colaboração de pesquisadores e estudantes (graduação e pós-graduação) da DFIS/UEFS,
LNA/MCTI 27] e IPD/UNIVAP [28]. Os objetos de estudo são as galáxias peculiares interagentes ESO 505-IG 030 e ESO 505-IG 031, classificadas como espirais do tipo Sc de acordo com o esquema de Hubble (ver Figura 1). Os espectros ópticos de fenda longa que fazem parte deste estudo foram obtidos no Observatório do Pico dos Dias (OPD/LNA-MCTI), associados aos projetos de pesquisa em desenvolvimento no DFIS/UEFS, através do GPGP (Grupo de Pesquisa em Galáxias Peculiares).

\section{GALÁXIAS PECULIARES: UMA RÁPIDA INTRODUÇÃ̃}

Com o crescente interesse nos objetos ditos "extragalácticos", impulsionados sobretudo pelos intrigantes resultados fotométricos e espectroscópicos obtidos, um outro astrônomo norte-americano inicia um estudo observacional com galáxias em processos de interação gravitacional, compilando, inicialmente, um catálogo de galáxias peculiares para o Hemisfério Norte [6] e, em seguida, um catálogo para o Hemisfério Sul [7. O principal objetivo dos catálogos foi o de revelar, através de milhares de imagens obtidas em chapas fotográficas, a existência de outros tipos de galáxias no Universo local (redshift $<0,1)$ com estruturas diferenciadas, ou seja, "peculiares". Como elemento motivador para tal estudo, estava a percepção de Halton Christian Arp (1927-2013) de que as galáxias com as estruturas morfológicas presentes na classificação de Hubble não eram as únicas e que o processo de formação e evolução destes objetos careciam de novas interpretações. Dentre as diversas análises feitas, ele percebeu que tais galáxias observadas, chamadas então de peculiares, representavam "laboratórios experimentais" para testes da dinâmica galáctica, onde os astrônomos poderiam utilizá-las para compreender processos físicos que ocorrem, não apenas no Universo local, mas também em larga escala.

A partir dos catálogos compilados, os astrônomos teriam em mãos uma primeira amostra de galáxias peculiares para um estudo 
mais detalhado envolvendo os resultados diretos de interações gravitacionais, que podem ser divididos, a grosso modo, em três tipos: (i) Colisão (collision), onde uma galáxia atravessa a outra, através de uma seção eficaz de impacto, (ii) Fusão (merging), onde dois objetos combinam-se em um único, e (iii) Efeito de Maré (tidal interaction), onde uma galáxia (projétil) passa próxima de uma outra (alvo) perturbando-a gravitacionalmente. Esse efeito tende a esticar os objetos na direção de aproximação e seguem três propriedades fundamentais [10]:

1. A força de maré é proporcional ao inverso do cubo da separação entre as galáxias;

2. As forças de maré sobre um objeto tende a alongá-lo; assim, os bojos de maré se formam no lado mais próximo e no lado mais distante de cada galáxia em relação à outra;

3. As galáxias perturbadas geralmente giram antes do encontro de maré e a distribuição posterior de seu material deve, portanto, refletir a conservação de seu momentum angular.

É esperado, em um processo de interação gravitacional entre duas ou mais galáxias participantes, que a dinâmica propicie a formação de estruturas diversas como pontes, caudas, laços, filamentos, jatos, anéis, detritos, etc., associado ao fato de que os respectivos movimentos orbitais se encontram em um plano que forma um ângulo qualquer com a nossa linha de observação.

As estruturas peculiares atualmente observadas foram cunhadas ao longo do tempo por meio dos diferentes processos gravitacionais mencionados acima. Embora existam dados observacionais (fotométricos e espectroscópicos) para a grande maioria desses objetos interagentes, não há, por outro lado, uma classificação geral para os mesmos, como vista na Figura 11, ou seja, que organize as diversas características morfológicas peculiares conhecidas até então. A resposta está no fato de haver inúmeras maneiras pelas quais duas (ou mais) galáxias podem afetar uma à outra, produzindo diferentes aspectos peculiares como descritos acima.
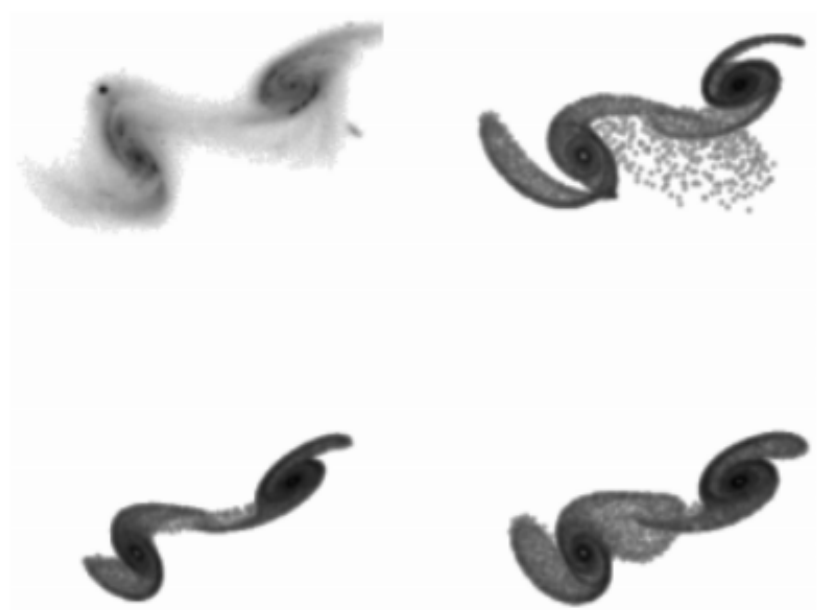

FIGURA 2: Parte de um modelo de simulação de N-corpos mostrando a interação gravitacional para a galáxia Arp 240. Fonte: referência [29].

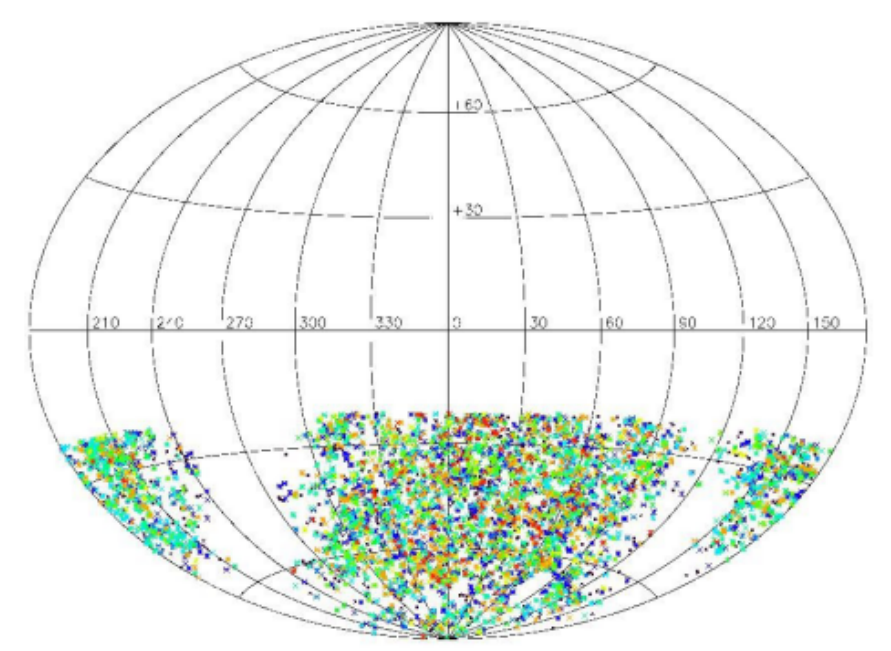

FIGURA 3: Distribuição em coordenadas equatoriais (projeção Aitoff) para as 6465 galáxias peculiares presentes no Catálogo [7]. Os pontos coloridos representam os objetos presentes em cada uma das 25 Categorias.

No entanto, uma separação por Categorias desses objetos (galáxias peculiares) pode ser proposta e foi realizada para as observações do Hemisfério Sul [7] que resultaram em A Catalogue of Southern Peculiar Galaxies and Associations. 
O Catálogo consiste de uma lista de 6445 objetos peculiares distribuídos em 25 Categorias, desde o pólo celeste Sul até a declinação de $-22^{\circ}$, registradas em um total de 537 placas fotográficas de $6,5^{\circ} \times 6,5^{\circ}$. O resultado da compilação encontra-se disponível em https://ned.ipac.caltech.edu/level5/SPGAAtlas/frames.html. A Figura 3 ilustra em coordenadas equatoriais (ascensão reta e declinação), todos os objetos presentes no Catálogo do Hemisfério Sul, enquanto a Figura 4 ilustra a frequência dos objetos em cada uma das 25 Categorias. A descrição é feita na Tabela VIII. presente no Apêndice VIII.

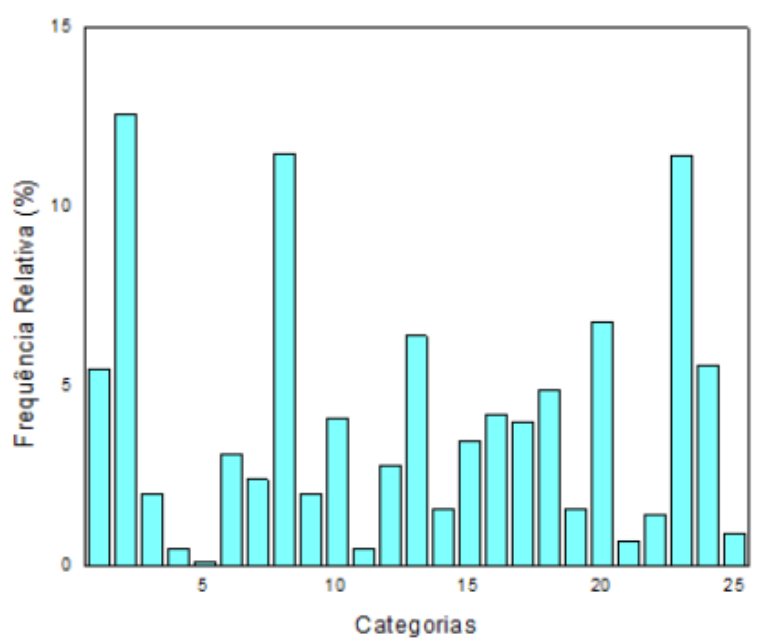

FIGURA 4: Frequência das galáxias peculiares por Categoria. Ver informação complementar na Tabela VIII.

\section{O PAR INTERAGENTE ESO 505-IG 030 E ESO 505-IG 031}

Uma análise detalhada que leve a uma compreensão mais robusta das intrigantes estruturas morfológicas observadas nas galáxias peculiares em processos de interação requer dados fotométricos e espectroscópicos em diferentes bandas (U,B,V,R,I) e comprimentos de onda. No entanto, acontece que tais objetos, quando comparados com aqueles presentes na classificação de Hubble, ainda são relativamente pouco estudados nas duas técnicas acima, dificultando, assim, em uma primeira aproximação, o conhecimento astrofísico intrínseco de um dado objeto e, em segunda, uma análise global, que poderia apontar possíveis correlações entre diversos parâmetros cinemáticos e dinâmicos para a amostra total de 6445 objetos.

Diante de tal realidade, o presente trabalho apresenta-se como um dos frutos que objetivam contribuir com resultados fotométricos e espectroscópicos que possam propiciar um melhor entendimento sobre a natureza nuclear e extranuclear das galáxias peculiares. Nesse sentido, selecionamos para o presente estudo o par de galáxias interagente ESO 505-IG 030 e ESO 505-IG 031, Figura 5, pertencente à Categoria 15 do Catálogo de Arp e Madore (1987) [7]: "Galáxias com Cauda, Laços de Matéria ou Detritos". A imagem ilustrada deste par (identificador 333 da placa fotográfica) foi publicada no Atlas de 1080 galáxias compilado por Vorontsov-Velyaminov em 1959.

De acordo com a classificação homogeneizada do NED/NASA-IPCA, tratam-se de duas galáxias espirais não barradas do tipo Sc, com magnitudes visuais na banda $\mathrm{R}$ (Cousins) de 13,98 \pm 0,09 mag (ESO 505-IG 030) e $14,23 \pm 0,09$ mag (ESO 505-IG 031), respectivamente. Em uma imagem de melhor resolução (Figura 6), é possível notar as estruturas de matéria menos luminosas que as regiões centrais. É importante ressaltar que na classe Sc das espirais, a região central é geralmente pequena, tendo nos braços uma contribuição bem maior para a luminosidade integrada. A maior parte destas galáxias apresentam braços múltiplos fracamente ligados à região central e facilmente resolvidos em regiões HII e associações estelares. É possível perceber também manchas de poeira interestelar que, em alguns casos, alcançam o núcleo.

As estruturas de matéria observada na Figura 6] são melhor caracterizadas como "caudas" ou "pontes" que podem facilmente ser interpretados como braços de galáxias espirais. No entanto, os braços múltiplos aparecem depois que os braços principais alcançam $90^{\circ}$ [13]. 


\section{MISSÃO OBSERVACIONAL}

O par interagente foi observado em 2013 com espectrógrafo Cassegrain (modo fenda longa) acoplado ao telescópio Perkin-Elmer de $1,6 \mathrm{~m}$ no OPD/LNA-MCTI. A Tabela VIII fornece alguns dados observacionais, enquanto a Tabela VIII apresenta alguns dos parâmetros instrumentais empregados, ambas estão no Apêndice VIII É importante ressaltar que as noites relativas à missão observacional não foram fotométricas, com o seeing da ordem de 1,5 segundos de arco.

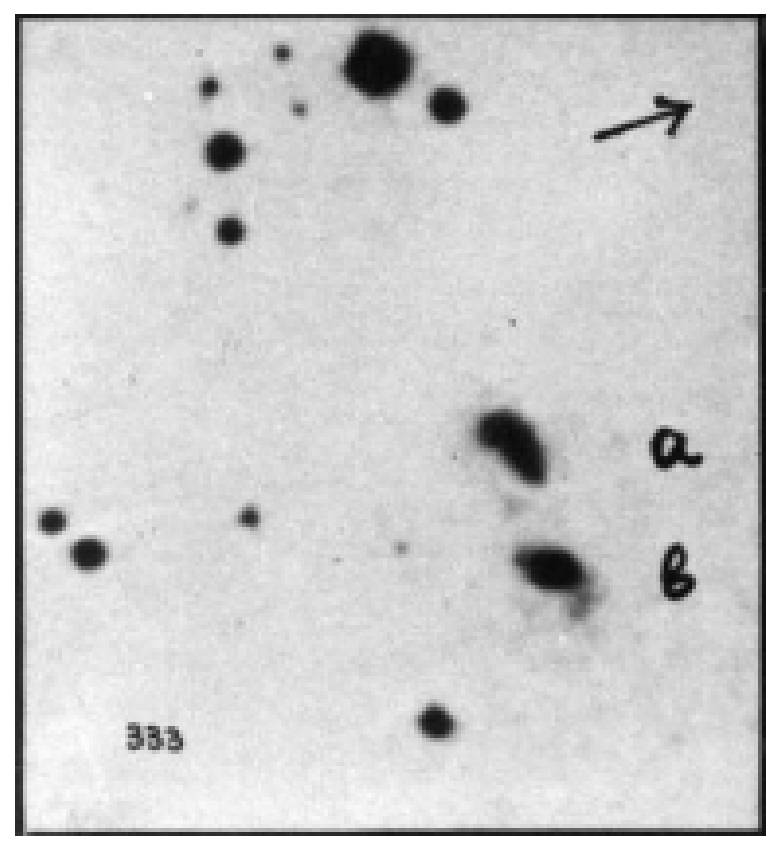

FIGURA 5: Imagem do par interagente ESO 505IG 030 (objeto b) e ESO 505-IG 031 (objeto a) presente na Categoria 15 do Catálogo de Arp e Madore (1987). A seta indica a direção do Norte.

As observações espectroscópicas foram realizadas com o espectrógrafo Cassegrain e o CCD IKON 17588 com $2048 \times 2048$ pixels $(13,5 \times 13,5) \mu \mathrm{m}$, sem redutor focal, o que fornece uma escala de placa de 0,18 segundos de arco por pixel. Os espectros foram obtidos no modo de baixa resolução com a rede de 300 linhas por mm, o que permitiu obter uma maior cobertura espectral $(\lambda 3380$ a $\lambda 8620 \AA$, centrada em $\lambda 6000 \AA$ ), incluindo as principais linhas de emissão observadas na região do visível (H $\alpha \lambda 6563 \stackrel{\circ}{A}$, [NII] $\lambda \lambda 6548,6584 \stackrel{\circ}{A}, \mathrm{H} \beta$ $\lambda 4861 \AA$, [OIII] $\lambda \lambda 4959,5007 \AA$ ) e também as linhas de absorção (banda $\mathrm{G}$ do $\mathrm{CH} \lambda 4301 \AA$, MgI $\lambda 5174 \AA$, MgH $\lambda 5269 \AA$, NaID $\lambda 5892 \AA$, TiO $\lambda 6250 \AA$ ). O ganho e o ruído de leitura calculados foram, respectivamente, em 0,9 elétrons/ADU e 6,9 elétrons. De acordo com a posição geométrica dos objetos no campo de observação, uma única posição de fenda (direção Leste-Oeste) foi usada ao longo do eixo maior de cada galáxia.

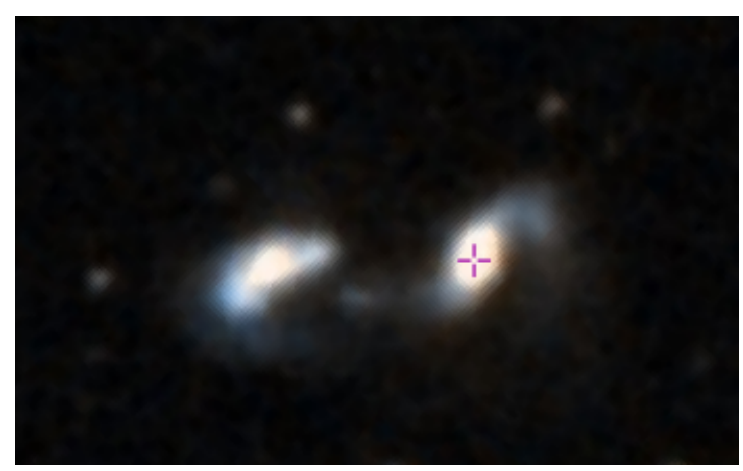

FIGURA 6: Recorte do par interagente ESO 505-IG 031 (lado esquerdo) e ESO 505-IG 030 (lado direito) extraído do Aladin Sky Atlas (https://aladin.u-strasbg.fr/AladinLite/). Norte para cima e Leste para a esquerda.

\section{ESTUDO CINEMÁTICO}

Em 1912, ao analisar a luz proveniente da galáxia M31 (Andrômeda), o astrônomo norte-americano Vesto Melvin Slipher (1875 1969) percebeu que as linhas espectrais estavam ligeiramente deslocadas para o azul, ou seja, para os menores comprimentos de onda quando comparadas aos espectros do referencial de repouso, i.e., do laboratório [30]. Em termos da frequência, dizemos que a luz assim observada era maior do que o valor esperado. No entanto, acontece que, no caso da luz ou do som, o resultado observado (associado ao efeito Doppler) é causado pelo movimento relativo entre a fonte e o observador e, 
nesse caso, indicava que ambos (fonte e observador) estavam se aproximando. A conclusão imediata que podemos extrair, pelo menos para estas duas galáxias (M31 e a Via Láctea), é que estas não se encontram fixas no Universo. Mas, além da dúvida deste resultado ser aplicado para as demais galáxias observadas, existe também aquela que interroga se todas estão mutuamente se aproximando, ou seja, se o Universo está passando por um regime de colapso. A rotação das galáxias espirais foi uma outra descoberta importante de Slipher [31].

A partir desses resultados e de algumas possíveis interpretações Slipher, após um longo período de estudo, descobriu que na amostragem observada, a grande maioria apresentava deslocamento espectral para o vermelho, isto é, para os maiores comprimentos de onda ou baixas frequências, indicando assim que elas estavam se afastando do observador. O resultado agora era outro e apontava para uma provável expansão do Universo, embora algumas delas apresentavam a conclusão obtida inicialmente para M31. A descoberta ainda apontava que, para as galáxias mais afastadas (de baixo brilho superficial), o deslocamento espectral observado para o vermelho era maior.

Alguns anos mais tarde, Hubble entra novamente em cena e publica em 1929 [32] e depois em 1931 com Milton La Salle Humason (18911972) 33, o trabalho que descreve as velocidades radiais de 46 nebulosas extragalácticas e as distâncias individuais para 24 destas. Os dados observacionais obtidos quando apresentados em um gráfico velocidade $(v)$ versus distância $(d)$ indicava que quanto mais distante está a galáxia, maior é a velocidade associada de afastamento, ou seja:

$$
v=H_{0} d,
$$

sendo $H_{0}$ a constante de Hubble. A Eq.(1) é chamada lei de Hubble. Na época, as distâncias medidas se mostraram superestimadas, fornecendo um valor inicial para a constante da ordem de $600 \mathrm{~km} / \mathrm{s} / \mathrm{Mpc}$, contaminada, provavelmente, por problemas na calibração da relação período-luminosidade e também por erros de identificação na amostra das estrelas Cefeidas usadas. Atualmente, o valor é consideravelmente menor, $68 \pm 2$ $\mathrm{km} / \mathrm{s} / \mathrm{Mpc}$ [34. Nesse estudo, usamos o valor de $73 \mathrm{~km} / \mathrm{s} / \mathrm{Mpc}$, já empregado em outros estudos do nosso grupo de pesquisa [35 41].

O desvio para o vermelho (redshift) de uma fonte astronômica, caracteriza o nome que comumente atribuímos ao deslocamento do espectro observado na direção daqueles de maiores comprimentos de onda em relação ao espectro observado no referencial de repouso da fonte. $\mathrm{O}$ redshift é representado pela letra $z$ e é expresso pela relação:

$$
z=\Delta \lambda / \lambda_{\text {rep }}=\left(\lambda_{r e p}-\lambda_{\text {obs }}\right) / \lambda_{\text {rep }},
$$

onde $\lambda_{\text {obs }}$ e $\lambda_{\text {rep }}$ são, respectivamente, os comprimentos de onda de um fóton medidos no referencial do observador e em repouso. O comprimento de onda observado é expresso, mediante a relação $c=\lambda \nu$, na forma:

$$
\lambda_{o b s}=\lambda_{r e p} \sqrt{\frac{1+\nu_{r} / c}{1-\nu_{r} / c}},
$$

onde $\nu_{r}=\nu \cos \theta$ é a componente da velocidade radial na direção do observador, $\theta$ representa o ângulo entre o vetor velocidade e a linha de visada. Deste modo, o redshift pode ser escrito:

$$
z=\sqrt{\frac{1+\nu_{r} / c}{1-\nu_{r} / c}}-1 .
$$

Em geral, a Eq. (2) quando combinada com a relação $\lambda=c / \nu$ revela que,

$$
z+1=\Delta t_{o b s} / \Delta t_{r e p},
$$

cuja interpretação pode ser feita da seguinte forma: se a luminosidade de uma fonte astrofísica que se afasta do observador $(z>0)$ variar durante um intervalo de tempo $\Delta t_{o b s}$, a mudança na luminosidade irá ocorrer em um intervalo de tempo mais curto, ou seja, no sistema de repouso da fonte $\Delta t_{r e p}=\Delta t_{o b s} /(z+1)$. $\mathrm{O}$ trabalho de Hubble ratifica, portanto, aquele de Slipher e é considerado uma evidência experimental da expansão do Universo. De fato, o desvio para o vermelho é detectável em todas as direções observáveis. 
Esta contribuição busca determinar os parâmetros físicos (redshift, velocidade heliocêntricas, distância, massa e metalicidade) do par interagente ESO 505-IG 030 e ESO 505IG 031, de forma a contribuir no estudo sobre a natureza peculiar desses objetos. Para isso, é necessário um estudo detalhado dos dados obtidos na missão observacional já citada, a partir da redução e síntese espectral (explicadas a seguir). Analisaremos, também, os parâmetros geométricos (elipticidade, excentricidade e inclinação) de ambas as galáxias a partir das imagens disponíveis na literatura.

\section{RESULTADOS OBTIDOS E DISCUSSÃO}

\section{A. Redução dos Dados}

A redução espectroscópica dos dados foi realizada através dos pacotes disponíveis no IRAF [42] (Image Reduction and Analysis Facility). Os procedimentos padrões seguidos foram: subtração do bias, normalização por flat-field, remoção dos raios cósmicos usando a técnica descrita em van Dokkum [43], extração de espectros unidimensionais e calibração em comprimento de onda e em unidades de fluxo. Uma descrição detalhada empregada nas reduções espectroscópicas para as galáxias peculiares estudadas no GPGP pode ser obtida na referência [37.

Realizamos para cada perfil espacial das galáxias estudadas um número suficiente de aberturas (janelas de extração - região nuclear e extranuclear) com o propósito de obter um razoável mapeamento ao longo da posição da fenda, alinhada ao longo do eixo-maior de cada galáxia. A partir do conjunto de espectros formados, calibrados posteriormente em fluxo e comprimento de onda, devidamente corrigidos da extinção Galáctica [44, foi possível inferir sobre a cinemática de cada galáxia estudada. Com a tarefa XCSAO presente no pacote RVSAO (A Radial Velocity Package) [45] no ambiente IRAF, determinamos as velocidades heliocêntricas das galáxias em questão, utilizando as aberturas centrais de cada galáxia.
Embora represente uma forma direta de obter o valor do redshift $z$, a técnica espectroscópica possui um considerável custo observacional quando se trata de objetos fracos (galáxias distantes com baixo brilho superficial) que necessitam um longo tempo de integração em telescópios de pequena abertura. Para os objetos estudados, o tempo total foi de $2400 \mathrm{~s}$ por objeto (ver Tabela VIII).

Para as galáxias ESO 505-IG 030 e ESO 505-IG 031, respectivamente, um total de 14 e 11 aberturas foram realizadas para cada perfil espacial ao longo da posição da fenda, alinhada na direção leste-oeste de cada galáxia - ver Figura 6. As variáveis necessárias para cada cálculo serão obtidas para cada espectro da janela de extração de 3 segundos de arco através do ambiente IRAF. A Figura 7 apresenta o mapeamento das diversas janelas de extração em cada perfil espacial.

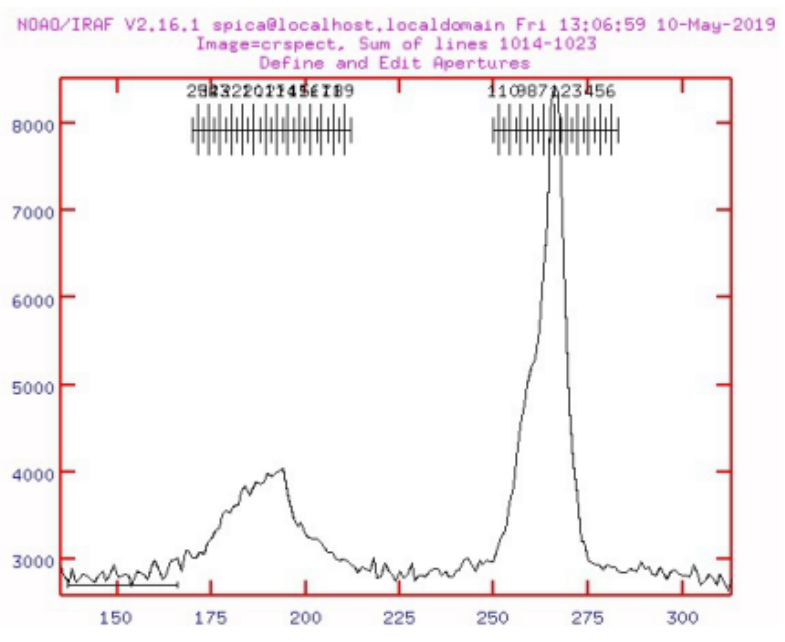

FIGURA 7: Aberturas feitas em cada perfil espacial ao longo da posição da fenda. Na Figura, a galáxia IG 030 encontra-se a esquerda e a galáxia IG 031 a direita.

\section{B. Parâmetros Físicos: velocidade, redshift e distância}

A partir dos pacotes presentes no IRAF, consegue-se determinar os seguintes parâmetros físicos, redshift $(z)$, velocidade heliocêntrica $(v)$ e a distância $(d)$ das galáxias 
em questão, utilizando a abertura central de cada objeto, devidamente calibrada em fluxo $\left(\mathrm{erg} / \mathrm{cm}^{2} / \mathrm{s}\right)$ e corrigida por extinção e efeito Doppler. A Tabela II apresenta os resultados obtidos neste trabalho e a comparação com os valores de referência publicados no NED/NASA-IPAC. Assumimos ao longo deste estudo a constante de Hubble igual a 73 $\mathrm{km} / \mathrm{s} / \mathrm{Mpc}$ [46].

A pequena diferença em nossos resultados para as velocidades e distâncias relativas dos dois objetos (aproximadamente $50 \mathrm{~km} / \mathrm{s}$ e 0,7 $\mathrm{Mpc}$ ) reforça a natureza interagente do par, associado ao processo de interação gravitacional por efeito de maré [47, 48]. Na maioria das galáxias vizinhas, as distâncias projetadas são da ordem de $\Delta V<100 \mathrm{~km} / \mathrm{s}$ para a velocidade e de $\Delta D<100 \mathrm{kpc}$ para a separação espacial 47. Os resultados estão em bom acordo com os publicados na literatura: IG 030 e IG 031 .

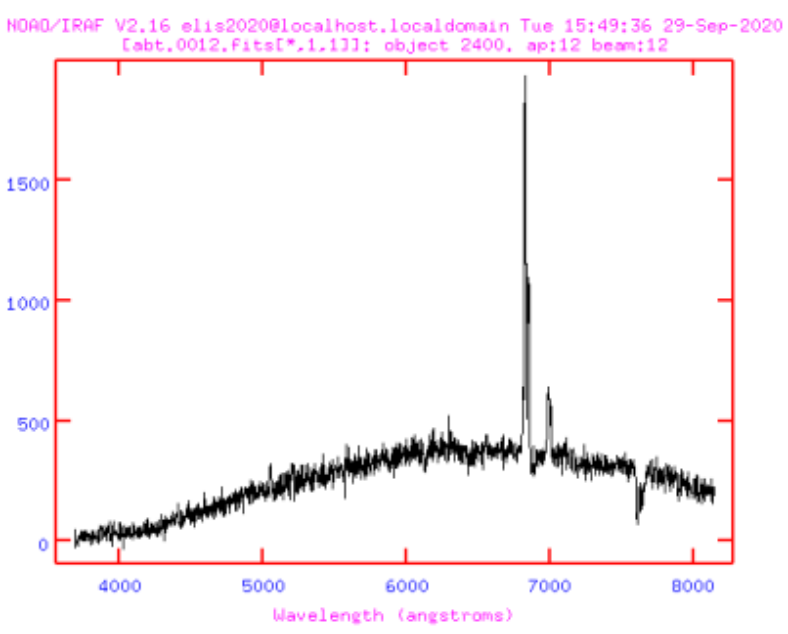

FIGURA 8: Espectro da abertura central da galáxia ESO 505-IG 030. Nota-se as linhas de emissão. Espectro calibrado apenas em comprimento de onda.

As velocidades determinadas para as galáxias podem ser interpretadas de duas maneiras: "peculiares", isto é, relacionadas com o movimento através do espaço e "recessionais", ou seja, relacionadas com a própria expansão do Universo, de acordo com a Lei de Hubble. Da mesma maneira, o "redshift cosmológico" de uma galáxia deve ser interpretado pela expansão quando o comprimento de onda da luz emitida pela mesma é "esticado" junto com o espaço por onde ela viaja. Por esta razão, o redshift cosmológico não está relacionado à velocidade de recessão da galáxia pelas equações do deslocamento Doppler (ver equações na seção V].

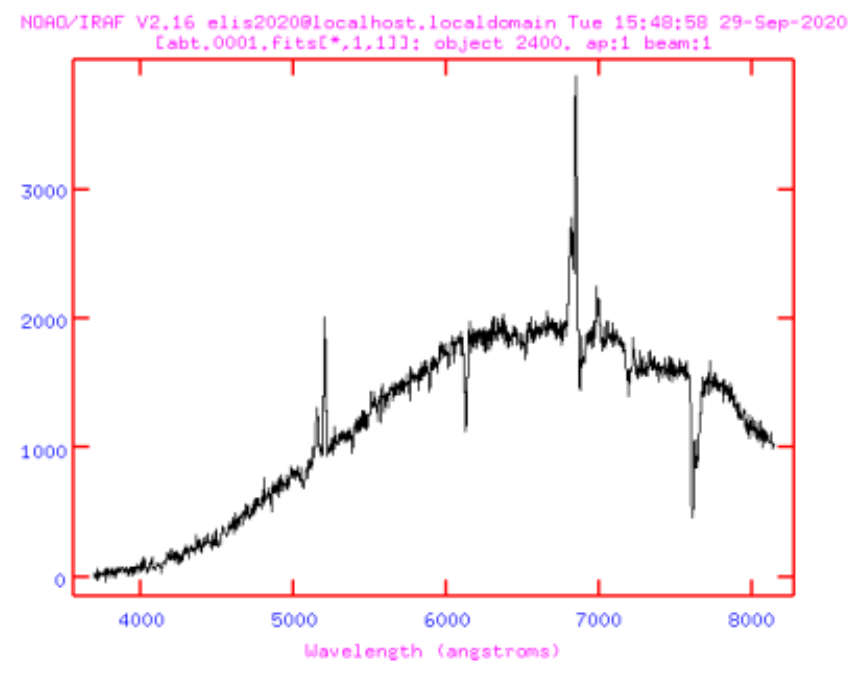

FIGURA 9: Espectro da abertura central da galáxia ESO 505-IG 031. Veja a descrição na Figura 8 .

Conceitualmente, as equações Doppler foram formuladas para um espaço-tempo Euclidiano, estático, e não incluem os efeitos da expansão e da curvatura do espaço-tempo. Ainda assim, usaremos frequentemente a Eq. (4) na forma:

$$
\frac{\nu_{r}}{c}=\frac{(z+1)^{2}-1}{(z+1)^{2}+1}
$$

para traduzir uma medida de redshift $z$ no lugar de uma velocidade radial que uma galáxia teoria. Em outras palavras, uma velocidade peculiar (movendo-se pelo espaço) ao invés de uma velocidade recessional atual (movendo-se junto com a expansão do espaço).

No que concerne às distâncias, para $z \leq$ 2 , os valores geralmente estimados através da Eq.(6) com a lei de Hubble em (1),

$$
d \simeq \frac{c}{H_{0}} \frac{(z+1)^{2}-1}{(z+1)^{2}+1},
$$


diferem das distâncias próprias atuais menos do que $5 \%$, assumindo um Universo do tipo "plano", isto é, um modelo cuja solução é favorecida pelo cenário inflacionário e pelas observações da radiação cósmica de fundo (veja [49]). Por outro lado, realizando uma expansão de primeira ordem no termo $\left(1+\nu_{r} / c\right)^{ \pm 1 / 2}$,

$$
\left(1+\nu_{r} / c\right)^{ \pm 1 / 2} \simeq 1+\frac{\nu_{r}}{2 c},
$$

e com auxílio das Equações (2) e (3), podemos escrever para baixas velocidades:

$$
z=\frac{\Delta \lambda}{\Delta \lambda_{\text {rep }}} \simeq \frac{\nu_{r}}{c}
$$

onde $\nu_{r}>0$ para uma fonte que se afasta $(\Delta \lambda>0)$ e $\nu_{r}<0$ para uma fonte que se aproxima $(\Delta \lambda<0)$. Portanto, quando $z \ll 1$, o estudo de caso deste trabalho, a expressão para a distância assume a forma não-relativística,

$$
d=\frac{c \nu}{H_{0}},
$$

e pode levar erros significativos na distância para valores da ordem de $z>0,4$. Os valores presentes na Tabela II, portanto, para $z \approx$ 0,04 , são representativos, válidos e encontramse no intevalo $\pm 1 \sigma$ em relação às medidas publicadas na literatura (ver Figura 10).

\begin{tabular}{ccc}
\hline ESO 505-IG & $c z_{O P D}$ & $c z_{\text {(literatura) }}$ \\
\hline 030 & $11944 \pm 17$ & $11906 \pm 32^{a}$ \\
& & $12006 \pm 45^{b}$ \\
& & $11808 \pm 56^{c}$ \\
\hline 031 & $11895 \pm 53$ & $11822 \pm 36^{d}$ \\
& & $11831 \pm 45^{b}$ \\
& & $11744 \pm 70^{a}$ \\
\hline Média & \pm 35 & $\pm 47,3$ \\
\hline
\end{tabular}

TABELA I: Velocidades radiais heliocêntricas sistêmicas. Comparações com outros estudos: (a) Spectroscopic observations of Arp-Madore interacting galaxies-II. Galaxies with tails, loops of material or debris. 51]. (b) The $6 d F$ Galaxy Survey Data Release 3. [52]. (c) The 2MASS Redshift SurveyDescription and Data Release. [53] (d) [54].

A Figura 10 mostra uma comparação direta dos desvios das medidas da $c z$ deste trabalho com os valores publicados na literatura (Tabela II). Observe que das seis comparações, apenas duas apresentam diferenças maiores que $1 \sigma$ (desvio padrão da diferença: $c z_{O P D}-c z_{\text {literatura }}$ ). Como as nossas medidas possuem uma dispersão dentro de $\pm 1 \sigma$, este resultado confirma que os dados obtidos neste trabalho são consistentes com a literatura.

\begin{tabular}{cccc}
\hline Galáxia & $\mathrm{v}(\mathrm{Km} / \mathrm{s})$ & $\mathrm{z}$ & $\mathrm{d}(\mathrm{Mpc})$ \\
\hline IG 030 & $11944 \pm 17$ & 0,040660 & 163,6 \\
Sekiguchi e & & & \\
Wolstencroft (1993) & $11906 \pm 32$ & 0,039714 & 163,1 \\
IG 031 & $11895 \pm 53$ & 0,040498 & 162,9 \\
N. da Costa et al. (1988) & $11822 \pm 36$ & 0,039434 & 161,9 \\
\hline
\end{tabular}

TABELA II: valores dos parâmetros físicos.

\section{Curva de Rotação}

Uma vez calculadas as velocidades a partir das várias aberturas extraídas nos perfis espaciais, é possível construir um gráfico da curva de rotação. i.e., a velocidade de rotação em função da distância galactocêntrica (Figuras 11 e 12. . A distância em Mpc é obtida a partir da razão entre a velocidade e o valor $H_{0}$ adotado. O resultado, quando dividido por 206264, 8 (valor que representa o número de segundos de arco em 1 radiano), permite expressar o resultado em parsec por pixel, ou seja, a escala que iremos encontrar para as distâncias em cada janela de extração de 3 segundos de arco (arcsec). Neste caso, para ambas galáxias, o valor foi próximo de $0,79 \mathrm{Kpc} / \operatorname{arcsec}$. As velocidades foram obtidas como o pacote externo RVSAO desenvolvido pelo Smithsonian Astrophysical Observatory Telescope Data Center, no qual calcula as velocidades radiais dos espectros usando correlação cruzada e técnicas de ajuste nas linhas de emissão [50].

A galáxia ESO 505-IG 030 apresenta um comportamento rotacional relativamente suave, sem grandes flutuações a partir da abertura central. Por outro lado, ESO 505-IG 031 já oferece um perfil diferenciado, reportado na literatura como $U$-shaped rotation profile, descrito em estudos envolvendo sistemas gravitacionalmente perturbados [55]60]. Este tipo 
de perfil é comum em galáxias elípticas fortemente interagentes, cuja interpretação física, dada por [61], revela um acoplamento de maré entre a órbita da companheira e as estrelas em rotação prógrada ressonante na galáxia cinematicamente distorcida [56, 62, 63].

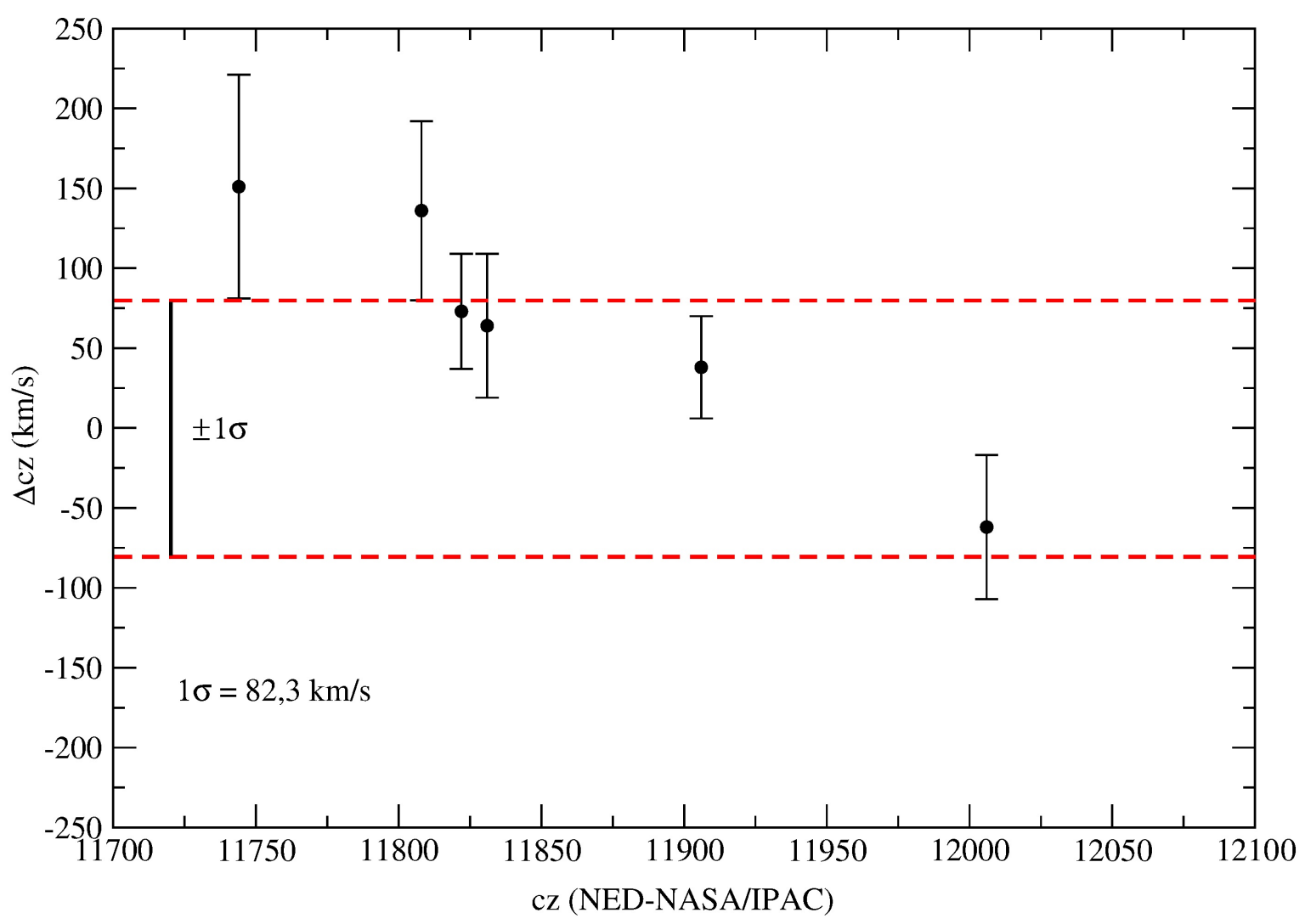

FIGURA 10: Diferenças entre as velocidades radiais heliocêntricas calculadas neste trabalho e as publicadas na literatura (NED-NASA/IPAC).

\section{Síntese Espectral: estudo da população estelar presente}

O código de síntese espectral STARLIGHT 64, 65] é um programa que modela o representativo da contribuição estelar do espectro observado e fornece um espectro sintetizado que melhor representa os espectros estudados em termos das populações estelares de diferentes idades e metalicidades (uma combinação linear de populações estelares simples, SSPs).

O objetivo consiste em modelar e remover a contribuição da população estelar subjacente, fornecendo, assim, um espectro residual (observado menos modelado), com apenas as linhas de emissão nebular, a exemplo daquelas descri- tas na subseção VIB

Usamos neste trabalho uma base de 150 elementos com 25 diferentes idades entre 1 Myr e 18 Gyr, e 6 metalicidades: $Z=0,005 ; 0,02 ; 0,2 ; 0,4 ; 1,0 ; 2,5$, somando 150 SSPs (veja 66 68]), extraídos dos modelos da referência [69], calculado para uma função de massa inicial [70] e trilhas evolutivas do tipo 'Padova (1994)' [71 75].

\section{Espectro Residual: linhas de emissão e absorção}

É possível notar, em ambos espectros (Figuras 13 e 14), as transições Balmer, $\mathrm{H} \alpha$ 
$(\lambda 6563 \AA)$ e $\mathrm{H} \beta(\lambda 4861 \AA)$, e as linhas de baixa ionização do [NII] $(\lambda \lambda 6548,6583 \AA$ A $)$ e do dubleto [SII] $(\lambda \lambda 6716,6730 \AA)$. É importante ressaltar a intensidade da linha do oxigênio duplamente ionizado [OIII] $(\lambda \lambda 4959,5007 \AA)$ presente apenas no espectro residual da galáxia IG 031, dominante em espectros de nebulosas planetárias. Ainda, temos a presença de uma fraca linha de emissão $\mathrm{H} \beta$ na galáxia IG 030 .

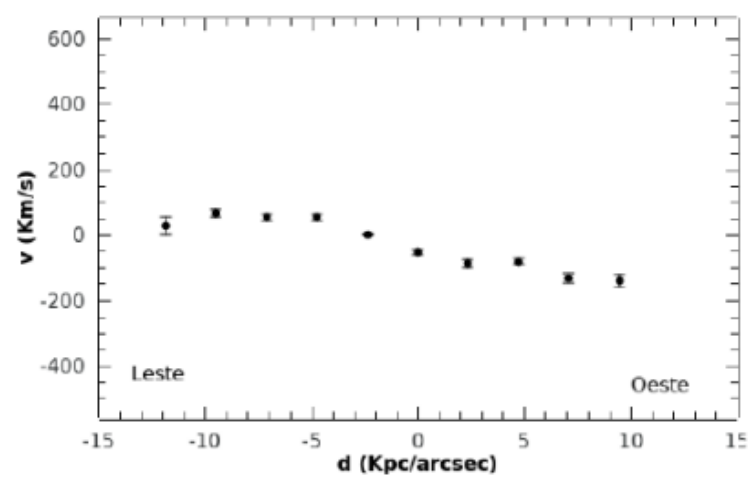

FIGURA 11: Gráfico da curva de rotação para a galáxia ESO 505-IG 030, com os respectivos erros determinados pelo pacote externo RVSAO no ambiente IRAF.

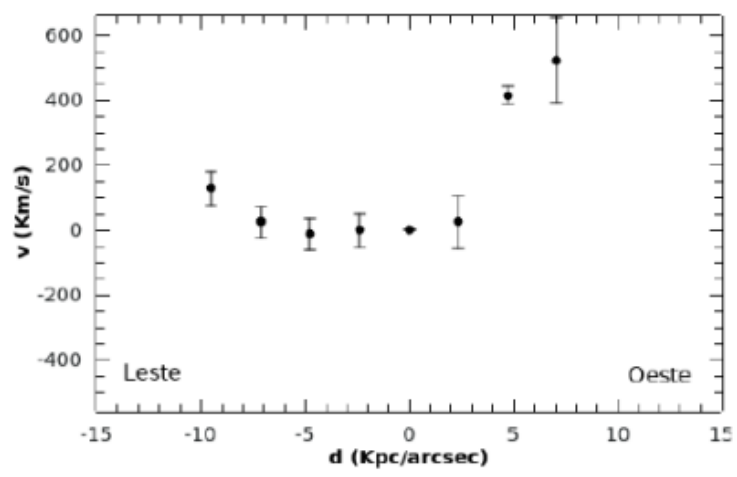

FIGURA 12: Gráfico da curva de rotação para a galáxia ESO 505-IG 031. Veja a descrição da Figura 11.

A absorção desta particular linha em ambas as galáxias, um obscurecimento de parte da região nuclear, pode ser causada por poeira ou por um aglomerado de estrelas não-ionizantes. Nota-se também a linha de absorção do NaID $(\lambda 5892 \AA)$ no espectro da galáxia IG 031, indicando a presença de estrelas do tipo espectral M. A dispersão observada na parte azul dos espectros está relacionada com a baixa eficiência quântica do detector empregado, inferior a $50 \%$.

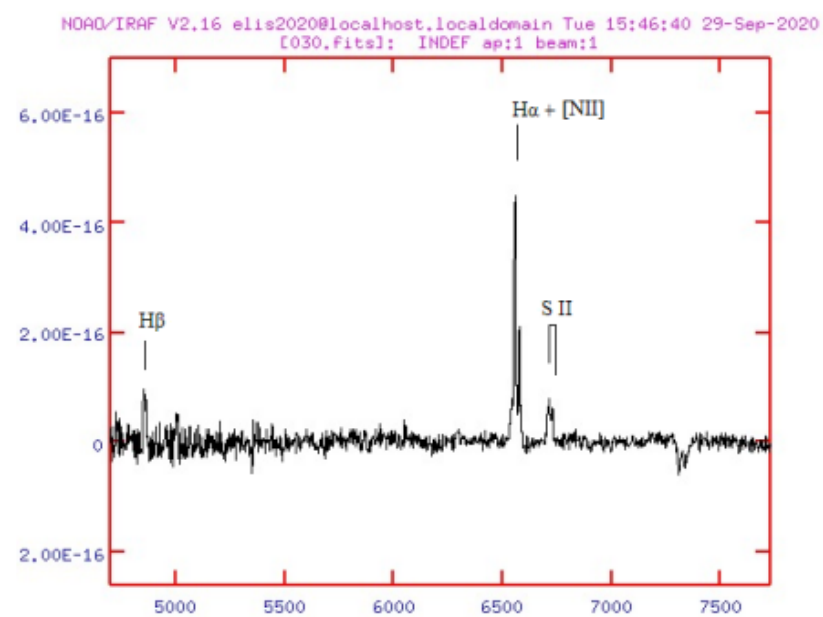

FIGURA 13: Espectro residual (observado menos modelado) da galáxia ESO 505-IG 030. Espectro calibrado em fluxo e comprimento de onda. As principais linhas de emissão estão representadas no espectro.

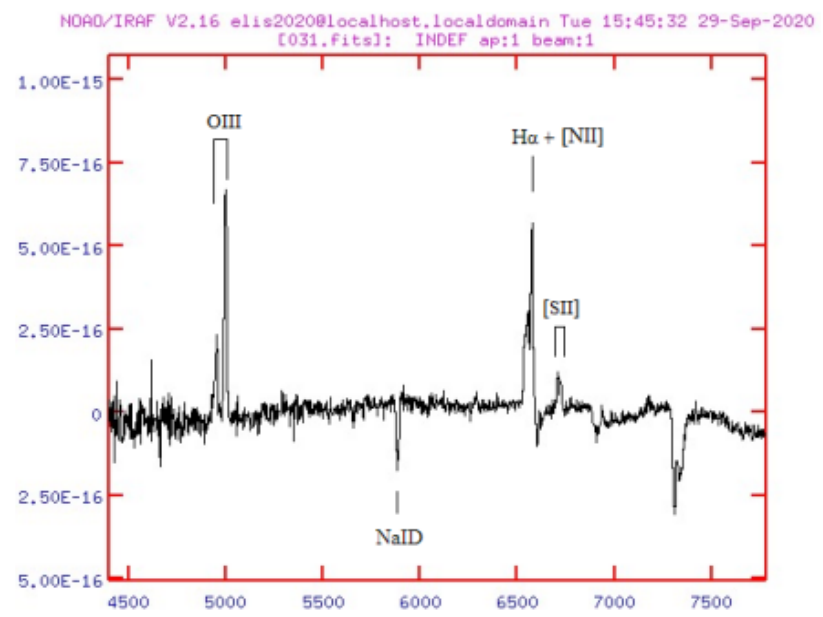

FIGURA 14: Espectro residual (observado menos modelado) da galáxia ESO 505-IG 031. Além das linhas de emissão, destacamos também a linha de absorção do NaID $\lambda 5892 \AA$. 


\section{População estelar, metalicidade e massa}

A síntese espectral também permite calcular as respectivas idades e as metalicidades das estrelas subjacentes presentes nos espectros observados. No caso das estrelas, adotamos o mesmo intervalo de representação presente em [65, ou seja: estrelas jovens $\left(t \leq 5 \times 10^{7}\right.$ anos), de idade intermediária $\left(5 \times 10^{7} \leq t \leq 2 \times 10^{9}\right.$ anos) e velhas $\left(2 \times 10^{9} \leq t \leq 13 \times 10^{9}\right.$ anos $)$. A síntese realizada forneceu os seguintes resultados percentuais para as estrelas assim definidas nos intervalos acima: $81 \%, 0 \%$ e $19 \%$ respectivamente, para a galáxia ESO 505-IG 030 e $35 \%, 59 \%$ e $6 \%$ para a ESO 505-IG 031.

Sabemos que as estrelas são constituídas, basicamente, de Hidrogênio e Hélio. Logo, as respectivas proporções dos outros elementos químicos, são, portanto, muito inferiores. $\mathrm{Na}$ Astronomia, denominamos de metais todos os elementos químicos, com exceção feito ao Hidrogênio e ao Hélio, embora muitos deles não sejam, de fato, metais. Desse modo, podemos dizer que uma certa estrela tem metalicidade alta se for "rica", por exemplo, em outros elementos químicos como Carbono e o Oxigênio. A metalicidade também pode ser estimada pelo código STARLIGHT, fornecendo, respectivamente para cada galáxia, $0,0046 Z_{\odot}$ para a galáxia ESO 505-IG 030 e $0,0029 Z_{\odot}$ para a galáxia ESO 505-IG 031. Nesta análise, adotamos para a metalicidade solar $\left(Z_{\odot}\right) 0,02$.

A distribuição de massa nas galáxias é uma aspecto crucial para as teorias que tratam da origem e de evolução dinâmica galáctica. Para as galáxias elípticas, as massas podem ser obtidas a partir das velocidades de dispersão das estrelas empregando o método do Virial. Por outro lado, a estimativa para as massas das galáxias estudadas, espirais do tipo Sc, devem ser obtidas a partir das curvas de rotação (ver Figuras 11 e 12, que fornecem a variação de suas respectivas velocidades com os raios. Assumindo que a maior parte da massa encontrase no bojo, a massa dentro do raio $R, M(R)$, pode ser estimada a partir da terceira lei de Kepler, através da equação,

$$
M(R)=R v(R)^{2} / G,
$$

onde $\mathrm{G}$ é a constante gravitacional equivalente a $6,67408 \times 10^{-11} \mathrm{~m}^{3} \mathrm{~kg}^{-1} \mathrm{~s}^{-2}$.

Nas regiões mais externas de muitas espirais, a velocidade $v(R)$ não depende de $R$, implicando que $M(R)$ é diretamente proporcional ao raio, ou seja, quanto mais longe se vai, maior é a quantidade de massa computada. A massa é obtida, inicialmente, em quilogramas, mas, para melhor analisar o resultado, é preferível estimar a massa em relação a massa solar $\left(\mathrm{M}_{\odot}\right)$, o que fornece, respectivamente para cada galáxia: $1,5 \times 10^{11} \mathrm{M}_{\odot}(\mathrm{ESO} 505-9 \mathrm{G} 030)$ e $9,2 \times 10^{10} \mathrm{M}_{\odot}($ ESO 505-IG 031).

\section{E. Parâmetros Geométricos}

Para completar o trabalho, fez-se interessante determinar os parâmetros geométricos (elipticidade, excentricidade e inclinação) dos objetos. No presente estudo, estes parâmetros foram estimados a partir das imagens fornecidas pelo banco de dados do Aladin Sky Atlas (http://aladin.u-strasbg.fr), com a devida escala de placa fornecida para a construção da relação entre pixels e segundos de arco.

Os parâmetros geométricos são geralmente determinados para as galáxias elípticas que compõem a classificação morfológica de Hubble, onde a geometria permite explorar os seguintes elementos: semi-eixos maior (a) e menor (b), razão axial $(b / a)$, elipticidade $(e)$, excentricidade $(\varepsilon)$ e ângulo de inclinação $(i)$. Uma vez calculados os semi-eixos maior para cada galáxia, os valores da elipticidade, excentricidade e a inclinação de cada galáxia (com respeito ao plano do céu observado) foram obtidos através das seguintes relações:

$$
\begin{aligned}
& e=1-b / a, \\
& \varepsilon=\frac{(a / b)^{2}-1}{(a / b)^{2}+1}, \\
& i=\cos ^{-1}(b / a) .
\end{aligned}
$$

Embora a morfologia resultante dos objetos interagentes não permita a determinação de tais parâmetros na maioria dos casos, as com- 


\begin{tabular}{ccccccc}
\hline Galáxia & $2 a$ & $2 b$ & $b / a$ & $e$ & $\varepsilon$ & $i$ \\
\hline IG 030 (banda V) & 26,25 & 14,41 & 0,55 & 0,44 & 0,55 & $56^{\circ} 46$ \\
Literatura (banda Ks)* & 27,00 & XX & 0,64 & 0,36 & 0,42 & $50^{\circ}$ \\
IG 031 (banda V) & 10,905 & 6,03 & 0,55 & 0,45 & 0,53 & $56^{\circ} 29$ \\
Literatura (banda Ks)* & 21,00 & XX & 0,60 & 0,40 & 0,47 & $53^{\circ}$ \\
\hline
\end{tabular}

TABELA III: Valores dos parâmetros geométricos das duas galáxias. Os eixos encontram-se nos valores de segundos de arco.

Fonte: ( ) 2MASS. Two Micron All Sky Survey Team (2MASS Extended objects) - Final release (2003).

ponentes do par de galáxias estudadas neste trabalho encontram-se em face-on, ou seja, projetadas com condições suficientes para que possam ser individualmente investigadas. Portanto, determinamos tais parâmetros neste trabalho e comparamos os mesmos com aqueles publicados na literatura.

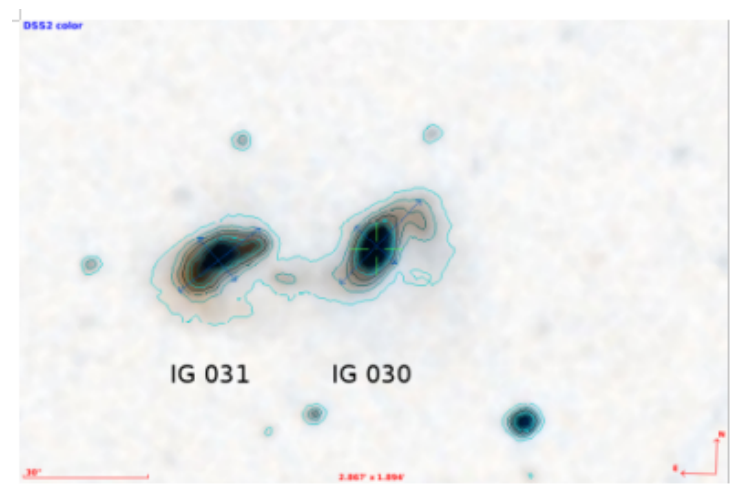

FIGURA 15: Par de galáxias interagentes ESO 505-IG 030 (Oeste) e ESO 505-IG 031 (Leste). Imagem obtida no banco de dados do Aladin Sky Atlas. Na imagem, a direção Norte é para cima, e a Leste para esquerda. As dimensões das imagens podem ser estimadas a partir da escala na barra do canto inferior esquerdo (30 segundos de arco).

A Figura 15 mostra os contornos realizados para o par de galáxias estudado, onde a escala de placa para a construção da relação entre pixel e segundos de arco vale: 1 pixel $=0,25$ arcsec. Para alcançar esses resultados, fizemos uso das equações descritas acima, presentes no banco de dados NED/NASA-IPAC. Portanto, empregando as ferramentas (contorno e distância) disponíveis no Aladin Sky Atlas, foi possível construir a Tabela III na qual fornece os valores estimados dos parâmetros para cada galáxia. Existe a perspectiva de obter imagens fotométricas dedicadas deste par de galáxias em uma próxima missão observacional no OPD/LNA-MCTI.

É importante salientar a presença de uma incerteza no resultado obtido, em particular, na definição da isófota (limite representativa de cada galáxia na banda do visível). Ainda, salientamos também que o estudo comparativo com a literatura (banda Ks, infravermelho) não foi realizado para a mesma banda fotométrica deste trabalho, pelo fato de não existir resultado na literatura, o que fornece uma outra análise fotométrica (isofotal). Ainda assim, os valores fornecem uma aproximação razoável para os tamanhos relativos de cada objeto.

\section{CONCLUSÕES}

O objetivo deste trabalho foi o de investigar, via espectroscopia de fenda longa, o par interagente ESO 505-9G 030 e ESO 505-9G 031, presente na Categoria 15 do Catálogo de [7]. Os espectros ópticos foram obtidos utilizando a rede de difração de 300 linhas por mm e empregando o espectrógrafo Cassegrain no telescópio de 1,60 $\mathrm{m}$ do OPD/LNA-MCTI.

A redução dos dados foi feita utilizando o software IRAF (Image Reduction and Analysis Facility), com um script previamente criado pelo grupo de pesquisa (GPGP). A partir do espectros reduzidos, devidamente corrigidos (extinção e efeito Doppler) e calibrados em fluxo, determinamos através do pacote RVSAO o redshift de cada galáxia, suas respectivas distânias e velocidades heliocêntricas. A diferença entre 
as velocidades heliocêntricas é menor que 100 $\mathrm{km} / \mathrm{s}$, com distância projetada de aproximadamente 0,7 Mpc, o que caracteriza, de fato, um sistema físico gravitacional interagente.

As curvas de rotação foram obtidas e ressaltamos, particularmente, a presença do $U$ shaped rotation profile, presente em sistemas interagentes. Porém, apenas as regiões mais centrais foram exploradas neste trabalho, tendo em vista uma forte dispersão nos valores das velocidades presente para $d>15 \mathrm{kpc} / \operatorname{arcsec}$, o que dificulta uma análise para regiões muito distantes da região nuclear. Para a galáxia ESO 505-IG 030, concluiu-se que a velocidade de rotação não diminui com a distância, mas se mantém praticamente constante a partir do disco. Para a galáxia ESO 505-IG 031, observamos que sua velocidade rotacional aumenta à medida que se afasta do núcleo, revelando que as caudas/laços de matéria giram com maior velocidade do que as regiões próximas ao disco, sendo mais uma indicação que existe interação gravitacional com a galáxia vizinha.

As idades e as metalicidades envolvidas foram fornecidas pela síntese espectral de população estelar, via código STARLIGHT [65], e os resultados obtidos estão em bom acordo com a morfologia dos objetos. As massas foram obtidas a partir das curvas de rotação, com os valores determinados de $1,5 \times 10^{11} \mathrm{M}_{\odot}(\mathrm{ESO}$ $505-9 \mathrm{G} 030)$ e $9,2 \times 10^{10} \mathrm{M}_{\odot}$ (ESO 505IG 031), dentro do intervalo esperado para galáxias espirais: $10^{9}$ a $10^{11} \mathrm{M}_{\odot}$.

Para completar o trabalho, utilizamos as imagens ópticas do banco de dados do Aladin Sky Atlas para inferir sobre a geometria das galáxias, i.e., calculamos valores de elipticidade, excentricidade e inclinação em relação a linha de visada do observador para ambos objetos, a partir das equações presentes no NED/NASA-IPAC. Os valores encontrados estão em bom acordo com a literatura, apesar do procedimento não garantir os limites isofotais corretos de cada galáxia. Outro aspecto está associado à banda espectral empregada em cada análise. Embora diferentes, (óptico, V) e (infravermelho, R), os resultados são satisfatórios para uma primeira análise desses parâmetros.

Por fim, existe a perspectiva de trabalharmos com a parte nuclear dos objetos, ou seja, a partir dos espectros residuais, investigar o tipo de atividade natureza presente. Outra perspectiva está associada a construção de um modelo computacional de $\mathrm{N}$-corpos que melhor representa a morfologia ora observada. Além do mais, fazer o estudo fotométrico em uma próxima missão observacional para adquirirmos resultados mais definitivos sobre a fotometria e geometria peculiar dessas galáxias.

\section{Agradecimentos}

Os autores agradecem a equipe técnica do OPD/LNA-MCTI pelo apoio durante as noites de observação vinculadas ao projeto OP2012ALP12, "Estudo Espectroscópico de Galáxias Peculiares e Associações". A autora principal agradece ao $\mathrm{CNPq}$ (Conselho Nacional de Desenvolvimento Científico e Tecnológico) pela bolsa de Iniciação Científica fornecida durante a graduação, já que a experiência é de extrema importância para o crescimento de novos pesquisadores. Os autores também agradecem à UEFS/PPPG (Universidade Estadual de Feira e Santana/Pró-Reitoria de Pesquisa e Pós-Graduação) e à FAPESB pelo apoio financeiro parcial para a realização desta pesquisa.

\section{APÊNDICE}

\begin{tabular}{cccccc}
\hline Galáxia & Data (dd/mm/aa) & Tempo Exposição (s) & PA (graus) & Massa de Ar Média S/N (1/A) \\
\hline ESO 505-IG 030 & $20 / 04 / 2013$ & 2400 & $90^{\underline{0}}$ (Leste-Oeste) & 1,182 & 55 \\
ESO 505-IG 031 & $20 / 04 / 2013$ & 2400 & $90^{\underline{0}}$ (Leste-Oeste) & 1,182 & 52 \\
\hline
\end{tabular}

TABELA IV: Algumas informações observacionais. P.A. representa o ângulo de posição da fenda de acordo com o arranjo instrumental fornecido pelo OPD/LNA-MCTIC. 


\begin{tabular}{ccc}
\hline Categoria & Breve Descrição & Frequência (\%) \\
\hline 1 & Galáxias com Companheiras & 5,5 \\
2 & Interagentes Duplas & 12.6 \\
3 & Interagentes Triplas & 2,0 \\
4 & Interagentes Quadruplas & 0,5 \\
5 & Interagentes Quíntuplas & 0,1 \\
6 & Galáxias Aneladas & 3,1 \\
7 & Galáxias com Jatos & 2,4 \\
8 & Galáxias com Companheiras Aparentes & 11,5 \\
9 & Tipo-M51 & 2,0 \\
10 & Galáxias com Braços Espirais Peculiares & 4,1 \\
11 & Galáxias com Três e Múltiplos Braços Espirais & 0,5 \\
12 & Galáxias com Discos Peculiares & 2,8 \\
13 & Galáxias Compactas & 6,4 \\
14 & Galáxias com Absorção não Usual de Poeira & 1,6 \\
15 & Galáxias com Caudas, Laços de Matéria ou Detritos & 3,5 \\
16 & Galáxias Irregulares & 4,2 \\
17 & Galáxias com Cadeias & 4,0 \\
18 & Galáxias em Grupos & 4,9 \\
19 & Galáxias em Aglomerados & 1,6 \\
20 & Galáxias Anãs & 6,8 \\
21 & Objetos Estelares com Nebulosas Associadas & 0,7 \\
22 & Miscelânia & 1,4 \\
23 & Pares Próximos & 11,4 \\
24 & Pares Triplos & 5,6 \\
25 & Nebulosas Planetárias & 0,9 \\
\hline
\end{tabular}

TABELA V: Frequência das galáxias peculiares nas diferentes Categorias.

\begin{tabular}{|c|c|c|}
\hline \multirow{4}{*}{ Observações } & Telescópio & Perkin-Elmer 1.6 m \\
\cline { 2 - 3 } & Observatório & Pico dos Dias (Brasópolis - MG) \\
\cline { 2 - 3 } & Espectrógrafo & Cassegrain (Boller \& Chivens) \\
\cline { 2 - 3 } & Rede & 300 linhas $/ \mathrm{mm}$ \\
\cline { 2 - 3 } & Dispersão & $0,5 \mathrm{~A}$ pixel-1 \\
\cline { 2 - 3 } & Resolução Espectral & $\sim 6,0 \mathrm{~A}(\mathrm{FWHM}): \sim 290 \mathrm{~km} / \mathrm{s}$ \\
\hline Calibração & He-Ar (lâmpada) & $? 6000 \mathrm{~A}(\mathrm{central})$ \\
\hline Detector & IKON 13739 & Resolução Temporal $(1 / 1000 \mathrm{~s})$ \\
\hline Amostragem Espacial & Tamanho do Pixel & $13,5 \times 13,5$ micrômetros \\
\hline Eficiência Quântica $(>90 \%$ no Visível e $<50 \%$ no Azul) & Matriz do CCD & $2048 \times 2048$ pixels \\
\hline
\end{tabular}

TABELA VI: Configuração instrumental. FWHM (Full Width at Half Maximum).

[1] Atualmente, sabemos que alguns daqueles objetos observados pertencem a nossa própria Galáxia, a Via Láctea, enquanto outros são, de fato, sistemas externos, ou seja, galáxias individuais.

[2] As Cefeidas clássicas, ou simplesmente Cefeidas, formam um dos grupos mais homogêneos e importantes de estrelas pulsantes, com períodos de 2 a 40 dias. Pertencem a população I (braços espirais) e o protótipo deste tipo é a estrela Delta Cephei, descoberta por John Goodricke (1764-1786) em 1784. São estrelas gigantes ou supergigantes amarelas, muito luminosas, cujas curvas de luz são regulares e com períodos bem definidos.

[3] Hoje, uma conhecida galáxia espiral localizada a cerca de 2,54 milhões de anos-luz de distância da Terra, na direção da constelação de Andrômeda.

[4] Na versão apresentada em 1936, a existência 
de galáxias do tipo S0 era ainda duvidosa.

[5] E. Hubble, The luminosity function of nebulae. I. The luminosity function of resolved nebulae as indicated by their brightest stars. The Astrophysical Journal 84, 158 (1936).

[6] H.C. Arp, Atlas of peculiar galaxies. The Astrophysical Journal Supplement Series 14, 1 (1966).

[7] H.C. Arp, B.F. Madore, B. Madore, A Catalogue of Southern Peculiar Galaxies and Associations: Positions and Descriptions. Vol. 1. Cambridge: Cambridge University Press (1987).

[8] J.E. Barnes, L. Hernquist, F. Schweizer, Colliding Galaxies. Scientific American 265, (2) 40 (1991).

[9] J.E. Barnes, Transformation of Galaxies. I. Mergers and Equal Mass Stellar Disk. ApJ 393, 484 (1992).

[10] J.E. Barnes, L. Hernquist, Dynamics of interacting galaxies. Annual Review of Astronomy and Astrophysics 30, (1) 705 (1992).

[11] J.E. Barnes, L. Hernquist, Computer Models of Colliding Galaxies. Physics Today 46, (3) 54 (1993).

[12] J. Binney, Warps. ARA\&A 30, 51 (1992).

[13] G. Bertin, Dynamics of Galaxies. Cambridge: Cambridge University Press (2000).

[14] J. Binney, S. Tremaine, Galactic Dynamics. New Jersey: Princeton University Press (1997).

[15] D. Mihalas, J. Binney, Galactic Astronomy: Structure and Kinematics. 2nd Edition. San Francisco: W.H. Freeman and Co. (1981).

[16] A. Dressler, S.M. Faber, D. Burstein, New velocity dispersions and photometry for $E$ and $S 0$ galaxies in the great attractor. The Astrophysical Journal 368, 54 (1991).

[17] T.M. Eneev, N.N. Kozlov, R.A. Sunyaev, A\&A 22, 4 (1973).

[18] W.C. Keel, Crashing Galaxies, Cosmic Fireworks. Sky and Telescope 77, (1) 18 (1989).

[19] W.C. Keel, The Real Astrophysical Zoo: Colliding Galaxies. Mercury 22, 44 (1993).

[20] R.B. Larson, Galaxy Building. Publications of the Astronomical Society of the Pacific 102, (653) 709 (1990).

[21] M.C. Schroeder, N.F. Comins, Galactic Collisions on your Computer. Astronomy 16, (12) 90 (1988).

[22] J. Silk, Formation of the Galaxies. Sky and Telescope 72, 582 (1986).

[23] J. Silk, The Big Bang. Revised and Updated Edition. New York: W.H. Freeman and Company (1989).
[24] A. Toomre, J. Toomre, Galactic bridges and tails. The Astrophysical Journal 178, 623 (1972).

[25] A. Toomre, J. Toomre, Violent Tides between Galaxies. Scientific American 229, (6) 38 (1973).

[26] S. van den Bergh, J.E. Hesser, How the Milky Way Formed. Scientific American 268, (1) 72 (1993).

[27] Laboratório Nacional de Astrofísica / Ministério de Ciência, Tecnologia e Inovação.

[28] Instituto de Pesquisa e Desenvolvimento / Universidade do Vale do Paraíba.

[29] A.J. Holincheck, et al., Galaxy Zoo: Mergers Dynamical models of interacting galaxies. MNRAS 459, (1) 720 (2016).

[30] V. Slipher, The radial velocity of the Andromeda Nebula. In: The Inaugural Volume of the Lowell Observatory Bulletin, 2.56 (1912).

[31] V. Slipher, The detection of nebular rotation. Lowell Observatory Bulletin, 62 (1914).

[32] E. Hubble, A relation between distance and radial velocity among extra-galactic nebulae. PNAS 15, (3) 168 (1929).

[33] E. Hubble, M.L. Humason, The velocitydistance relation among extra-galactic nebulae. The Astrophysical Journal 74, 43 (1931).

[34] G. Paturel, P. Teerikorpi, Y. Baryshev, Hubble law: measure and interpretation. Foundations of Physics 47, (9) 1208 (2017).

[35] P.C. da Rocha-Poppe, M. Faúndez-Abans, V.F. Fernandes-Martin, I.F. Fernandes, M. de Oliveira-Abans, A. Rodrígues-Ardila. MNRAS 4021480 (2010).

[36] P.C. da Rocha-Poppe, M. Faúndez-Abans, V.F. Fernandes-Martin, M. de Oliveira-Abans, I.F. Fernandes, P.J.A. Lago. MNRAS $4 \mathbf{4 0}$ 1145 (2011).

[37] P.C. da Rocha-Poppe, M. Faúndez-Abans, V.F. Fernandes-Martin, M. de Oliveira-Abans, G.A. Silva, P. Freitas-Lemes, C. Lima-Dias. MNRAS 488, 3685 (2019).

[38] M. Faúndez-Abans, A.C. Krabbe, M. de Oliveira-Abans, P.C. da Rocha-Poppe, I. Rodrigues, V.A. Fernandes-Martin, I.F. Fernandes. A\&A 543, A64 (2012).

[39] M. Faúndez-Abans, M. de Oliveira-Abans, A.C. Krabbe, P.C. da Rocha-Poppe, V.A. Fernandes-Martin, I.F. Fernandes. A\&A 558, A13 (2013).

[40] M. Faúndez-Abans, V.P. Reshetnikov, M. de Oliveira-Abans, A.C. Krabbe, P.C. da RochaPoppe, V.A. Fernandes-Martin, E.B. Amôres, P. Freitas-Lemes. A\&A 574, A70 (2015).

[41] P. Freitas-Lemes, A.C. Krabbe, M. Faúndez- 
Abans, P.C. da Rocha-Poppe, I. Rodrigues, M. de Oliveira-Abans, V.A. Fernandes-Martin. MNRAS 468, 3159 (2017).

[42] IRAF é um sistema de software de uso geral para a redução e análise de dados astronômicos. O IRAF é distribuído pelo National Optical Astronomical Observatories (NOAO), que é operado pela Associação de Universidades para Pesquisa em Astronomia (AURA), Inc. sob contrato com a NSF. Homepage http://iraf.noao.edu/.

[43] P.G. van Dokkum, Cosmic-Ray Rejection by Laplacian Edge Detection. The Publications of the Astronomical Society of the Pacific 113, (789) 1420 (2001).

[44] D.J. Schlegel, textitet al., Maps of Dust Infrared Emission for Use in Estimation of Reddening and Cosmic Microwave Background Radiation Foregrounds. The Astrophysical Journal 500, (2) 525 (1998).

[45] Página da Internet - http://tdcwww.harvard.edu/iraf/rvsao/ .

[46] W.L. Freedman, B.F. Madore, The Hubble Constant. Annual Review of Astronomy and Astrophysics 48, 673 (2010).

[47] C-Y Kuo, J. Lim, Y-W Tang, P.T.P. Ho. ApJ 679, (2) 1047 (2008).

[48] Y.-W. Tang, C.-Y. Kuo, J. Lim, P.T.P. Ho. ApJ 679, (2) 1094 (2008).

[49] J.A. Peacock, Cosmological Physics. Cambridge: Cambridge University Press (2012).

[50] D.J. Mink, M.J. Kurtz, RVSAO 2.0-A radial velocity package for IRAF. Astronomical Data Analysis Software and Systems VII 145, (1998).

[51] K. Sekiguchi, R.D. Wolstencroft, Spectroscopic observations of Arp-Madore interacting galaxies-II. Galaxies with tails, loops of material or debris. Monthly Notices of the Royal Astronomical Society 263, (2) 349 (1993).

[52] D.H. Jones, et al., The $6 d F$ Galaxy Survey Data Release 3. MNRAS 399, (2) 683 (2009).

[53] J. Huchra, et al., The 2MASS Redshift SurveyDescription and Data Release. ApJS 199, 26 (2012).

[54] L.N. da Costa, et al, The southern sky redshift survey. The Astronomical Journal 116, (1) 1 (1998).

[55] K.D. Borne, Tidal Phenomena in Interacting Galaxies. In: R. Wielen (Editor), Dynamics and Interactions of Galaxies. Berlin: Springer (1990).
[56] K.D. Borne, J.G. Hoessel. BAAS 17, 601 (1985).

[57] K.D. Borne, J.G. Hoessel. ApJ 330, 51 (1988).

[58] R. Bender, A.Paquet, J.-L. Nieto. A\&A 246, 349 (1991).

[59] R. Madejsky. A\&A 247, 348 (1991).

[60] R. Madejsky, R. Bender, C. Möllenhoff. A\&A 242, 58 (1991).

[61] K.D. Borne, M. Balcells, J.G. Hoessel, M. McMaster. ApJ 435, 79 (1994).

[62] K.D. Borne. ApJ 330, 38 (1988).

[63] M. Bacells, K.D. Borne, J.G. Hoessel. ApJ 336, 655 (1989).

[64] Página da Internet http://www.starlight.ufsc.br/

[65] R.C. Fernandes, et al., Semi-empirical analysis of Sloan Digital Sky Survey galaxies-I. Spectral synthesis method. Monthly Notices of the Royal Astronomical Society 358, (2) 363 (2005).

[66] A. Mateus, et al., Semi-empirical analysis of Sloan Digital Sky Survey galaxies - II. The bimodality of the galaxy population revisited. MNRAS 370, (2) 721 (2006).

[67] N.V. Asari, R.C. Fernandes, et al., The history of star-forming galaxies in the Sloan Digital Sky Survey. MNRAS 381, (1) 263 (2007).

[68] R.C. Fernandes, N.V. Asari, et al., Uncovering the chemical enrichment and mass-assembly histories of star-forming galaxies. MNRAS 375, (1) L16 (2007).

[69] A.G. Bruzual, S. Charlot. MNRAS 344, 1000 (2003).

[70] G. Chabrier. ApJ 586, 133 (2003).

[71] M. Alongi, G. Bertelli, A. Bressan, C. Chiosi, F. Fagotto, L. Greggio, E. Nasi. A\&AS 97, 851 (1993).

[72] A. Bressan, F. Fagotto, G. Bertelli, C. Chiosi. A\&AS 100, 647 (1993).

[73] F. Fagotto, A. Bressan, G. Bertelli, C. Chiosi. A\&AS 104, 365 (1994).

[74] F. Fagotto, A. Bressan, G. Bertelli, C. Chiosi. A\&AS 105, 29 (1994).

[75] L. Girardi, A. Bressan, C. Chiosi, G. Bertelli, E. Nasi. A\&AS 117, 113 (1996).

[76] A. Dekel, S. Joseph, The origin of dwarf galaxies, cold dark matter, and biased galaxy formation. The Astrophysical Journal 303, 39 (1986).

[77] R.F.G. Wyse, J. Silk, Star formation rates and abundance gradients in disk galaxies. The Astrophysical Journal 339, 700 (1989). 